\title{
An efficient user scheduling scheme for downlink Multiuser MIMO-OFDM systems with Block Diagonalization
}

\author{
Mounir Esslaoui and Mohamed Essaaidi \\ Information and Telecommunication Systems Laboratory \\ Abdelmalek Essaadi University \\ Tetouan, Morocco
}

\begin{abstract}
The combination of multiuser multiple-input multiple-output (MU-MIMO) technology with orthogonal frequency division multiplexing (OFDM) is an attractive solution for next generation of wireless local area networks (WLANs), currently standardized within IEEE 802.11ac, and the fourth-generation (4G) mobile cellular wireless systems to achieve a very high system throughput while satisfying quality of service $(\mathrm{Q} O S)$ constraints. In particular, Block Diagonalization (BD) scheme is a low-complexity precoding technique for MU-MIMO downlink channels, which completely pre-cancels the multiuser interference. The major issue of the BD scheme is that the number of users that can be simultaneously supported is limited by the ratio of the number of base station transmit antennas to the number of user receive antennas. When the number of users is large, a subset of users must be selected, and selection algorithms should be designed to maximize the total system throughput. In this paper, the BD technique is extended to MU-MIMO-OFDM systems and a low complexity user scheduling algorithm is proposed to find the optimal subset of users that should transmit simultaneously, in light of the instantaneous channel state information (CSI), such that the total system sum-rate capacity is maximized. Simulation results show that the proposed scheduling algorithm achieves a good trade-off between sum-rate capacity performance and computational complexity.
\end{abstract}

Keywords-MU-MIMO; OFDM; scheduling; precoding; Block Diagonalization;

\section{INTRODUCTION}

The forthcoming breed of wireless standards, commonly referred to as fourth generation (4G) systems (LTE-Advanced, WiMAX or IEEE 802.11ac), are expected to satisfy the increasing demand for high data rates while satisfying quality of service (QoS) constraints. In particular, multiple-input multiple-output (MIMO) technology has been also acknowledged as one of the most promising techniques to achieve dramatic improvement in physical-layer (PHY) performance [1], [2]. While orthogonal frequency division multiplexing (OFDM) has long been regarded as an efficient approach to mitigate the effects of inter-symbol interference (ISI) in frequency-selective channels by dividing the entire channel into many narrow parallel sub-channels [3]. Therefore, the combination of these two technologies, which is called MIMO-OFDM, is an efficient way for providing high data rate reliable communications [4].

It is well known that multiuser MIMO (MU-MIMO) techniques are capable of significantly increasing capacity compared to traditional MIMO wireless systems [5]. Precoding techniques greatly influence the performance of MU-MIMO transmission by increasing the system capacity and/or reducing the complexity of the receiver when channel state information (CSI) is available at the transmitter [6]. It is assumed that the sum-rate capacity of MIMO broadcast channels can be achieved by applying dirty paper coding (DPC) at the transmitter [7]. Unfortunately, implementing this technique in practice is still a challenging task because of the complicated encoding and decoding schemes [8], especially when the number of users is large. To avoid the complexity of DPC, there have been a lot of interests in developing low-complexity precoding methods.

Block Diagonalization (BD) is an alternative and more practical precoding technique for downlink broadcast MU-MIMO channel and considered as an extension of zero forcing beamforming (ZFBF) for multiantenna receivers [9]. In particular, BD transforms the MU-MIMO downlink channel into parallel single-user MIMO (SU-MIMO) channels and completely nulls all interference between users without inverting the channel. However, this nulling operation imposes a constraint that the number of users that can be simultaneously supported with $\mathrm{BD}$ is limited by the ratio of the number of base station (BS) transmit antennas to the number of user receive antennas [9], which is not always feasible. Hence, user scheduling becomes necessary. Optimal scheduling method involves searching through all possible combinations of user subsets. However, as the number of users grows, the size of the search space becomes computationally very complex. To reduce this complexity, a large number of existing user selection approaches (e.g., [10], [11], [12]) has been employed to achieve a sum-rate capacity close to the one promised by DPC. Unfortunately, these frameworks solely targets single-carrier architectures. 


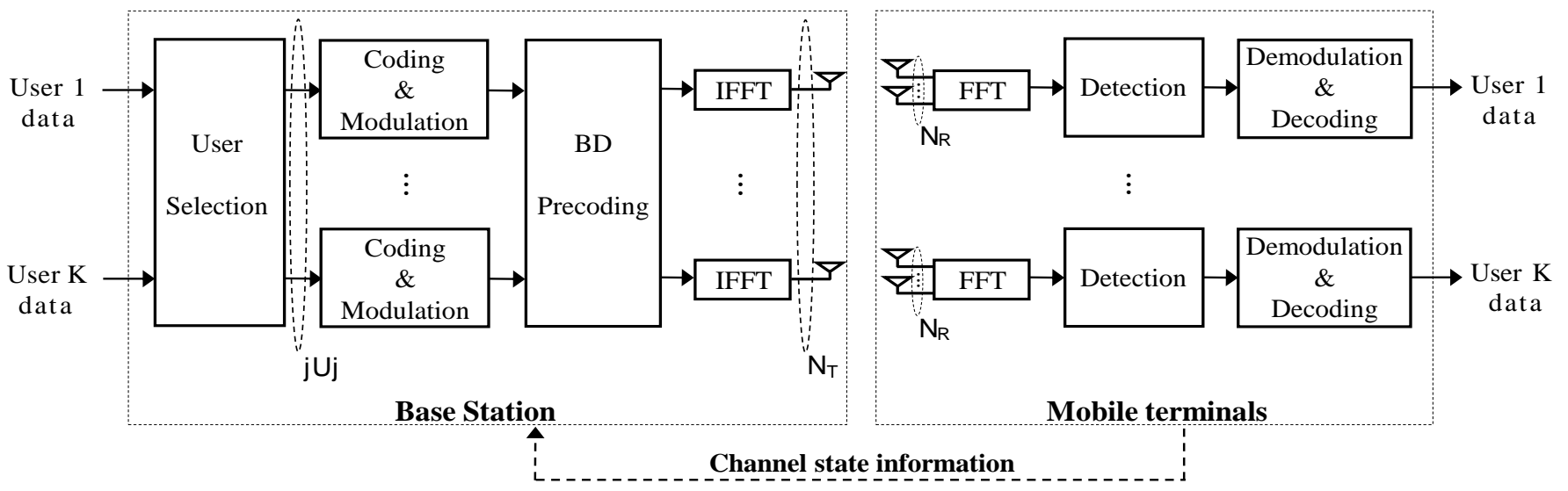

Fig. 1. Block diagram of MU-MIMO-OFDM downlink system with $N_{T}$ transmit antennas and $K$ users, each with $N_{R}$ receive antennas

Recently, authors in [13] proposed a low-complexity user selection algorithm for multicarrier networks based on OFDM, where the simultaneously transmitting users must share the utilization of all the subcarriers in the system (i.e., frequency is not used for multiple access as in OFDMA systems). Nevertheless, the work in [13] was limited to single-antenna receivers. In this paper, the $\mathrm{BD}$ technique for inter-user interference pre-cancelation is extended to MU-MIMO-OFDM systems and a low-complexity scheduling algorithm, based on [13], is developed considering the availability of multiple-antenna receivers. Numerical results show that the proposed scheme aim to select a subset of users such that the total system throughput is nearly maximized and close to the one obtained by the complex exhaustive search algorithm.

The remainder of this paper is organized as follows: Section II introduces the system model. Block Diagonalization for MU-MIMO-OFDM is presented in Section III. Scheduling algorithms for MU-MIMO-OFDM are described in Section IV and the numerical results are shown in Section V. Finally, Section VI summarizes the main outcomes of the paper and provides hints for further work.

\section{- Notational remark}

Boldface letters denote matrix-vector quantities while non-bold letters are used for scalars. The operation $(.)^{\mathrm{T}}$ and $(.)^{\mathrm{H}}$ represent the transpose and the Hermitian transpose of a matrix, respectively, and $\|\mathbf{A}\|_{\mathrm{F}}^{2}$ denote the Frobenius-norm (F-norm) of a matrix A. $|\mathcal{U}|$ is the cardinality of subset $\mathcal{U}$, $\mathbb{E}($.$) denotes the expectation operator, \operatorname{Tr}($.$) is the trace and \mathbb{C}$ is the set of complex numbers.

\section{SYSTEM MODEL}

Consider the downlink of a MU-MIMO-OFDM system, as illustrated in Fig. 1, with a single base station (BS) equipped with $N_{T}$ transmit antennas and $K \geq N_{T}$ geographically dispersed mobile users, each equipped with $N_{R}$ receive antennas. The system operates in a total bandwidth $W$ that is exploited by means of $N_{c}$ OFDM subcarriers. The BS broadcasts to all $K$ users simultaneously over all OFDM subcarriers. Each user $k$ receives from the base station $L_{k}$ data streams on every subcarrier with $L_{k} \leq N_{R}$, resulting in a total of $N_{c} L_{k}$ data streams per user. Hence, the BS transmits a total of $N_{c} \sum_{k=1}^{K} L_{k}$ data streams over all the $N_{c}$ parallel subcarriers to all the $K$ mobile terminals.

The data streams vector on subcarrier $q, \mathbf{s}_{k}[q] \in \mathbb{C}^{N_{R} \times 1}$, contains the data symbols of user $k$. The $N_{T} \times 1$ overall data vector of transmitted symbols from the BS antennas on subcarrier $q$ for all $K$ users is

$$
\mathbf{x}[q]=\left[\mathbf{s}_{1}^{\mathrm{T}}[q] \mathbf{s}_{2}^{\mathrm{T}}[q] \cdots \mathbf{s}_{K}^{\mathrm{T}}[\mathrm{q}]\right]^{\mathrm{T}} .
$$

The received signal at the $k$ th user on subcarrier $q$ for an arbitrary OFDM symbol is given by

$$
\mathbf{y}_{k}[\mathrm{q}]=\mathbf{H}_{k}[q] \mathbf{x}[q]+\mathbf{n}_{k}[q], \quad k=1, \ldots, K
$$

where $\mathbf{H}_{k}[q] \in \mathbb{C}^{N_{R} \times N_{T}}$ represents the channel gain matrix corresponding to the $k$ th user over the $q$ th subcarrier and $\mathbf{n}_{k}[q]$ is a zero-mean circularly symmetric additive complex gaussian noise with covariance matrix $\mathbf{R}_{\eta}=\sigma_{\eta}^{2} \mathbf{I}_{N_{R}}$. The transmitted signal is subject to an average power constraint $P_{T}$, which implies $\operatorname{Tr}\left(\mathbb{E}\left(\mathbf{x}[q] \mathbf{x}[q]^{\mathrm{H}}\right)\right) \leq P_{T}$.

Let $\mathcal{U}=\left\{u_{1}, \ldots, u_{|u|}\right\} \quad\left(|\mathcal{U}| \leq N_{T} / N_{R}\right)$ denote a set of users to whom the BS, in light of the available CSI, simultaneously transmits data during a given time slot. Each user's data stream, on subcarrier $q$, is preprocessed at the transmitter with the precoding matrix $\mathbf{W}_{k}[q] \in \mathbb{C}^{N_{T} \times N_{R}}$, so that the transmitted signal is a linear function that can be written as

$$
\mathbf{x}[q]=\sum_{k=1}^{|u|} \mathbf{W}_{k}[q] \mathbf{s}_{k}[q],
$$

and thus, the resulting received signal vector for user $k$ on subcarrier $q$ may be rewritten as

$$
\begin{aligned}
\mathbf{y}_{k}[q] & =\sum_{j=1}^{|u|} \mathbf{H}_{k}[q] \mathbf{W}_{j}[q] \mathbf{s}_{j}[q]+\mathbf{n}_{k}[q] \\
& =\mathbf{H}_{k}[q] \mathbf{W}_{k}[q] \mathbf{s}_{k}[q]+\sum_{j=1, j \neq k}^{|u|} \mathbf{H}_{k}[q] \mathbf{W}_{j}[q] \mathbf{s}_{j}[q]+\mathbf{n}_{k}[q] \\
& =\mathbf{H}_{k}[q] \mathbf{W}_{k}[q] \mathbf{s}_{k}[q]+\mathbf{c}_{k}[q]+\mathbf{n}_{k}[q],
\end{aligned}
$$

where the term $\mathbf{c}_{k}[q]$ in (6) corresponds to the multi-user interference that represents the major impairment in this scenario.

Transmission strategies for MU-MIMO-OFDM downlink channels are characterized by how we treat the multi-user interference $\mathbf{c}_{k}[q]$. The availability of channel knowledge at 
both ends of the link allows the transmitter to design the precoding matrices to pre-cancel the interference before transmission. Block Diagonalization (BD), proposed in [9] for single-carrier systems, is a precoding technique based on the orthogonalization of the signals that completely eliminate all multiuser interference followed by the waterfilling algorithm [14] to maximize the sum-rate capacity. An extension of the BD approach to multicarrier architecture based on OFDM systems is described in the next section.

\section{Block Diagonalization For MU-MIMO-OFDM}

The key idea of the Block Diagonalization approach is to find the precoding matrices $\left\{\mathbf{W}_{k}[q]\right\}_{k}^{|u|}$ for each user $k$ on all subcarriers to pre-eliminate the multiuser interference such that

$$
\mathbf{H}_{k}[q] \mathbf{W}_{j}[q]=0, \forall j \neq k .
$$

This decomposes the MU-MIMO channel into a set of parallel single-user MIMO (SU-MIMO) channels. Hence the received signal (6) for user $k$ on subcarrier $q$ can be simplified to

$$
\mathbf{y}_{k}[q]=\mathbf{H}_{k}[q] \mathbf{W}_{k}[q] \mathbf{s}_{k}[q]+\mathbf{n}_{k}[q] .
$$

Define the aggregate channel matrix and precoding matrix, on subcarrier $q$, for all the selected users in the subset $\mathcal{U}$, respectively, as

$$
\begin{aligned}
& \mathbf{H}_{u}[q]=\left[\begin{array}{lll}
\mathbf{H}_{1}^{\mathrm{T}}[q] \mathbf{H}_{2}^{\mathrm{T}}[q] \cdots & \mathbf{H}_{|\mathcal{T}|}^{\mathrm{T}}[q]
\end{array}\right]^{\mathrm{T}} \\
& \mathbf{W}_{u}[q]=\left[\begin{array}{lll}
\mathbf{W}_{1}[q] \mathbf{W}_{2}[q] \cdots \mathbf{W}_{|u|}[q]
\end{array}\right] .
\end{aligned}
$$

The zero-interference constraint (7) makes the product $\mathbf{H}_{\mathcal{U}}[q] \mathbf{W}_{\mathcal{u}}[q]$ block diagonal and forces $\mathbf{W}_{k}[q]$ to lie in the null space of the matrix $\overline{\mathbf{H}}_{k}[q]$ defined as [9]

$$
\overline{\mathbf{H}}_{k}[q]=\left[\mathbf{H}_{u_{1}}^{T}[q] \cdots \mathbf{H}_{u_{k-1}}^{T}[q] \mathbf{H}_{u_{k+1}}^{T}[q] \cdots \mathbf{H}_{u_{|u|}}^{T}[q]\right]^{T} .
$$

This constraint allows us to define the dimension condition necessary to guarantee that all users can be accommodated. The condition guarantees that data can be transmitted to user $k$, on the $q$ th subcarrier, if the null space of $\overline{\mathbf{H}}_{k}[q]$ has a dimension greater than 0 . This is satisfied when $\operatorname{rank}\left(\overline{\mathbf{H}}_{k}[q]\right)<N_{T}$. So for any $\mathrm{H}_{u}[q]$, block digitalization is possible if

$$
\max \left\{\operatorname{rank}\left(\overline{\mathbf{H}}_{u_{1}}[q]\right) \cdots \overline{\mathbf{H}}_{u_{|u|}}[q]\right\}<\mathrm{N}_{\mathrm{T}} .
$$

Assuming the dimension condition is satisfied for all users, let $L_{k}[q]$ be the rank of $\overline{\mathbf{H}}_{k}[q]$ and let the singular value decomposition (SVD) of $\overline{\mathbf{H}}_{k}[q]$ for each user $k \in \mathcal{U}$, on subcarrier $q$, be defined as

$$
\overline{\mathbf{H}}_{k}[q]=\overline{\mathbf{U}}_{k}[q] \overline{\boldsymbol{\Sigma}}_{k}[q]\left[\overline{\mathbf{V}}_{k}^{1}[q] \overline{\mathbf{V}}_{k}^{0}[q]\right]^{H}
$$

where $\overline{\mathbf{U}}_{k}[q]$ and $\overline{\mathbf{\Sigma}}_{k}[q]$ are the left singular vector matrix and the matrix of singular values of $\overline{\mathbf{H}}_{k}[q]$, respectively. $\overline{\mathbf{V}}_{k}^{1}[q]$ contains the first $L_{k}[q]$ right singular vectors and $\overline{\mathbf{V}}_{k}^{0}[q]$ holds the last $\left(N_{T}-L_{k}[q]\right)$ right singular vectors.
Thus, $\overline{\mathbf{V}}_{k}^{0}[q]$ constitutes an orthogonal basis for the null space of $\overline{\mathbf{H}}_{k}[q]$. To satisfy the zero-interference constraint, the precoding matrix can be chosen as

$$
\mathbf{W}_{k}[q]=\overline{\mathbf{V}}_{k}^{0}[q] \mathbf{A}_{k}[q],
$$

where $\mathbf{A}_{k}[q]$ is the $L_{k}[q] \times N_{R}$ transmit beamformer matrix for user $k$ on subcarrier $q$. By doing so, the downlink system reduces to $|\mathcal{U}|$ parallel non-interfering single user MIMO channels, where the equivalent independent channel after precoding for the $k$ th user on the $q$ th subcarrier is expressed as

$$
\widetilde{\mathbf{H}}_{k}[q]=\mathbf{H}_{k}[q] \overline{\mathbf{V}}_{k}^{0}[q] .
$$

Hence, $\mathbf{A}_{k}[q]$ can be viewed as a transmit beamformer matrix on $\widetilde{\mathbf{H}}_{k}[q]$ for user $k$ on subcarrier $q$ and can be found through the SVD of the projection of the channel of the $k$ th user on the null space of $\overline{\mathbf{H}}_{k}[q]$, resulting in the product $\mathbf{H}_{k}[q] \overline{\mathbf{V}}_{k}^{0}[q]$. The SVD of the product is expressed as

$$
\mathbf{H}_{k}[q] \overline{\mathbf{V}}_{k}^{0}[q]=\mathbf{U}_{k}[q]\left[\begin{array}{ll}
\boldsymbol{\Sigma}_{k}[q] & 0 \\
0 & 0
\end{array}\right]\left[\mathbf{V}_{k}^{1}[q] \mathbf{V}_{k}^{0}[q]\right]^{H},
$$

where $\boldsymbol{\Sigma}_{k}[q]$ is a $\tilde{L}_{k} \times \tilde{L}_{k}$ matrix of singular values of $\mathbf{H}_{k}[q] \overline{\mathbf{V}}_{k}^{0}[q]$, with $\tilde{L}_{k}=\operatorname{rank}\left(\mathbf{H}_{k}[q] \overline{\mathbf{V}}_{k}^{0}[q]\right) . \mathbf{V}_{k}^{1}[q]$ is the matrix that holds the first $\tilde{L}_{k}$ right singular vectors of $\mathbf{H}_{k}[q] \overline{\mathbf{V}}_{k}^{0}[q]$. The product of $\overline{\mathbf{V}}_{k}^{0}[q]$ and $\mathbf{V}_{k}^{1}[q]$ now produces an orthogonal basis of dimension $\tilde{L}_{k}$ and represents the transmission vectors that maximize the information rate for user $k$ on subcarrier $q$ subject to producing zero interference. The beamformer matrix $\mathbf{A}_{k}[q]$ can hence be written as

$$
\mathbf{A}_{k}[q]=\mathbf{V}_{k}^{1}[q] \boldsymbol{\Lambda}^{1 / 2}[q],
$$

where $\boldsymbol{\Lambda}[q]$ is a diagonal power loading matrix, on the $q$ th subcarrier, whose entries are the allocated powers whose optimal values are obtained by the waterfilling algorithm [14] applied to the diagonal elements of the matrix $\Sigma[q]$ that is expressed as

$$
\Sigma[q]=\left[\begin{array}{lll}
\boldsymbol{\Sigma}_{1}[q] & & \\
& \ddots & \\
& & \boldsymbol{\Sigma}_{\mid \mathcal{}}[q]
\end{array}\right] .
$$

For each user $k \in \mathcal{U}$ on subcarrier $q$, the precoding matrix is then

$$
\mathbf{W}_{k}[q]=\overline{\mathbf{V}}_{k}^{0}[q] \mathbf{V}_{k}^{1}[q]
$$

Thus, the aggregate precoding matrix on each subcarrier $q$ becomes

$\mathbf{W}_{u}[q]=\left[\overline{\mathbf{V}}_{1}^{(0)}[q] \mathbf{V}_{1}^{1}[q] \overline{\mathbf{V}}_{2}^{0}[q] \mathbf{V}_{2}^{1}[q] \cdots \overline{\mathbf{V}}_{|u|}^{0}[q] \mathbf{V}_{|u|}^{1}[q]\right] \boldsymbol{\Lambda}^{1 / 2}[q]$.

The sum-rate capacity of the system resulting from BD over all subcarriers is expressed as (21) and (22), shown at the bottom of the page.

$$
\mathcal{R}_{\mathrm{BD}}(\mathcal{U})=\max _{\mathbf{W}_{u}[q], \mathbf{H}_{k}[q] \mathbf{W}_{j}[q]=0, j \neq k} \frac{1}{N_{c}} \sum_{q=1}^{N_{c}} \log _{2}\left|\mathbf{I}+\frac{1}{\sigma_{\eta}^{2}} \mathbf{H}_{u}[q] \mathbf{W}_{u}[q] \mathbf{W}_{u}^{\mathrm{H}}[q] \mathbf{H}_{u}^{\mathrm{H}}[q]\right|
$$




$$
=\max _{\mathbf{H}_{k}[q] \mathbf{W}_{j}[q]=0, j \neq k} \frac{1}{N_{c}} \sum_{q=1}^{N_{c}} \sum_{k=1}^{|u|} \log _{2}\left|\mathbf{I}+\frac{1}{\sigma_{\eta}^{2}} \mathbf{H}_{k}[q] \mathbf{W}_{k}[q] \mathbf{W}_{k}^{\mathrm{H}}[q] \mathbf{H}_{k}^{\mathrm{H}}[q]\right| .
$$

The waterfilling algorithm maximizes the system's sum-rate capacity. With $\mathrm{W}_{u}[\mathrm{q}]$ chosen as in (14), the sum-rate capacity of the BD method in (22) becomes

$$
\mathcal{R}_{\mathrm{BD}}(\mathcal{U})=\max _{\Lambda[q]} \frac{1}{N_{c}} \sum_{q=1}^{N_{c}} \log _{2}\left|\mathbf{I}+\frac{\Sigma^{2}[\mathrm{q}] \Lambda[q]}{\sigma_{\eta}^{2}}\right|,
$$

where this maximization is subject to the power constraint $P_{T}$. The block diagonalization technique applied is summarized in Algorithm 1.

\section{Algorithm 1 : Block Diagonalization algorithm. \\ 1: For $q=1 \ldots N_{c}$ and $k=1 \cdots|\mathcal{U}|$, compute SVD of $\overline{\mathrm{H}}_{k}[q]$.

$$
\overline{\mathbf{H}}_{k}[q]=\overline{\mathbf{U}}_{k}[q] \overline{\boldsymbol{\Sigma}}_{k}[q]\left[\overline{\mathbf{V}}_{k}^{1}[q] \overline{\mathbf{V}}_{k}^{0}[q]\right]^{\mathrm{H}} .
$$

2: Compute the SVD of the projection of $\mathbf{H}_{k}[q]$ on the right null space of $\overline{\mathbf{H}}_{k}[q]$

$$
\mathbf{H}_{k}[q] \overline{\mathbf{V}}_{k}^{0}[q]=\mathbf{U}_{k}[q]\left[\begin{array}{cc}
\boldsymbol{\Sigma}_{k}[q] & 0 \\
0 & 0
\end{array}\right]\left[\mathbf{V}_{k}^{1}[q] \mathbf{V}_{k}^{0}[q]\right]^{\mathrm{H}} .
$$

3: Use the waterfilling algorithm on the diagonal elements of $\boldsymbol{\Sigma}[q]$ to determine the optimal power loading matrix $\boldsymbol{\Lambda}_{k}[q]$ under the power constraint $P_{T}$.

4: Set the aggregate precoding matrix on each subcarrier $q$ as

$$
\mathbf{W}_{u}[q]=\left[\overline{\mathbf{V}}_{1}^{0}[q] \mathbf{V}_{1}^{1}[q] \overline{\mathbf{V}}_{2}^{0}[q] \mathbf{V}_{2}^{1}[q] \cdots \overline{\mathbf{V}}_{|u|}^{0}[q] \mathbf{V}_{|u|}^{1}[q]\right] \Lambda^{1 / 2}[q] .
$$

\section{SCHEDULING ALGORITHMS FOR MU-MIMO-OFDM}

\section{A. Optimal scheduling algorithm}

Let $K_{\max }=\left\lceil N_{T} / N_{R}\right\rceil$ be the maximum number of simultaneously selected users at a given time slot, where $\lceil$.$\rceil is$ the ceiling function. When $K$ is large, there are possibly many supportable subsets $\mathcal{U}$ that satisfy the dimensionality constraint, i.e., $|\mathcal{U}| \leq K_{\max }$. Consider the sum-rate problem; the multiuser diversity gain is achieved by selecting an optimal subset of users that maximizes the sum-rate capacity in (23). Mathematically, the optimal sum-rate capacity of the BD scheme with multiuser diversity is given by

$$
\mathcal{R}_{\mathrm{BD}}^{\mathrm{opt}}(\mathcal{U})=\max _{\substack{U \subset\{1,2, \ldots, K\} \\ 1 \leq|\mathcal{U}| \leq K}} \mathcal{R}_{\mathrm{BD}}
$$

Therefore, the optimal scheduling requires an exhaustive search through all $\sum_{i=1}^{K_{\max }}\left(\begin{array}{l}K \\ i\end{array}\right)$ possible combinations of subsets of simultaneously supported users and is computationally very complex especially when the number of users is large. Thus, significant research efforts have been made to find suboptimal low-complexity algorithms that can achieve a significant fraction of the best performance provided by a full search method. In this paper, we propose a low-complexity multicarrier user selection algorithm for BD.

\section{B. Multicarrier user selection algorithm for $B D$}

The multicarrier user selection algorithm for BD (MUS-BD), summarized in Algorithm 2, selects a subset $\mathcal{U}$ of users, up to $K_{\max }$, from a total user pool $\Omega_{1}=\{1,2, \ldots, K\}$ such that the sum-rate capacity (23) is maximized for a given time slot. The base station will then simultaneously transmit to all users in $\mathcal{U}$ using all the OFDM subcarriers of the system. The algorithm works as follows: The step (I) initializes counters and the user pool $\Omega_{1}$ with the total $K$ active users in the cell. In step (II.a), the matrix $\mathbf{G}_{k}[q]$ defined as

$$
\mathbf{G}_{k}[q]=\mathbf{H}_{k}[q]-\sum_{j=1}^{i-1} \mathbf{H}_{k}[q] \widehat{\mathbf{V}}_{u_{i}}^{\mathrm{H}_{i}^{\mathrm{H}}}[q] \widehat{\mathbf{V}}_{u_{i}}^{1}[q],
$$

is found as the component of the channel matrix $\mathbf{H}_{k}[q]$, for each user $k$ on the $q$ th subcarrier, orthogonal to the subspace spanned by $\left\{\mathbf{G}_{u_{1}}[q] \cdots \mathbf{G}_{u_{i-1}}[q]\right\}$ where $\left\{u_{1} \cdots u_{i-1}\right\}$ denote the indexes of the previously selected users. Step (II.b) selects the best user $u_{i}$, at each iteration $i$, the one with the maximum squared F-norm of its user channel component $\mathbf{G}_{k}$ averaged over all subcarriers, that as,

$$
u_{i}=\underset{k \in \Omega_{\mathrm{i}}}{\operatorname{argmax}} \frac{1}{N_{c}} \sum_{q=1}^{N_{c}}\left\|\mathbf{G}_{k}[q]\right\|_{\mathrm{F}}^{2} .
$$

\section{Algorithm 2 : Multicarrier user selection algorithm for BD \\ (I) Initialization:

$$
\Omega_{1}=\{1,2, \ldots, K\} ; K_{\max }=\left\lceil\frac{N_{T}}{N_{R}}\right\rceil ; \mathcal{U}=\emptyset ; i=1
$$ \\ (II) Main Loop: \\ while $\left(\left|\Omega_{\mathrm{i}}\right| \neq 0\right) \&\left(|\mathcal{U}|<K_{\max }\right)$ \\ (a) Orthogonality measure computation: \\ for Each user $k \in \Omega_{\mathrm{i}}$ do \\ for Each subcarrier $q$ do

$$
\mathbf{G}_{k}[q]=\mathbf{H}_{k}[q]-\sum_{j=1}^{i-1} \mathbf{H}_{k}[q] \widehat{\mathbf{V}}_{u_{i}}^{1^{H}}[q] \widehat{\mathbf{V}}_{u_{i}}^{1}[q]
$$ \\ end for \\ end for}

(b) Orthogonal user selection

$$
\begin{aligned}
& u_{i}=\underset{k \in \Omega_{\mathrm{i}}}{\operatorname{argmax}} \frac{1}{N_{c}} \sum_{q=1}^{N_{c}}\left\|\mathbf{G}_{k}[q]\right\|_{\mathrm{F}}^{2} \\
& \mathcal{U}=\mathcal{U} \cup\left\{u_{i}\right\} ; \Omega_{\mathrm{i}}=\Omega_{i} \backslash\left\{u_{i}\right\}
\end{aligned}
$$

(c) Singular value decomposition:

for Each subcarrier q do

$\mathbf{G}_{u_{i}}[q]=\widehat{\mathbf{U}}_{u_{i}}[q] \widehat{\boldsymbol{\Sigma}}_{u_{i}}[q]\left[\widehat{\mathbf{V}}_{u_{i}}^{1}[q] \widehat{\mathbf{V}}_{u_{i}}^{0}[q]\right]^{H}$

\section{end for}

(d) Intermediate user grouping:

$$
\begin{aligned}
& \Omega_{i+1}=\left\{k \in \Omega_{i}, k \notin U: \frac{1}{N_{c}} \sum_{q=1}^{N_{c}} \frac{\left\|\mathbf{H}_{k}[q] \widehat{\mathbb{V}}_{u_{i}}^{H}[q]\right\|_{\mathrm{F}}^{2}}{\left\|\mathbf{H}_{k}[q]\right\|_{F}^{2}\left\|\widehat{\mathbb{v}}_{i}^{1}[q]\right\|_{\mathrm{F}}^{2}}<\alpha\right\} \\
& i=i+1
\end{aligned}
$$

(III) Output: The selected user subset $u$ 
Then the user $u_{i}$ is included in the subset $\mathcal{U}$ of selected users and discarded from the total user pool $\Omega_{i}$. Singular value decomposition of the matrix $\mathbf{G}_{u_{i}}[q]$ is computed in step (II.c) for the selected user $u_{i}$ at the $i$ th iteration over all subcarriers as,

$$
\mathbf{G}_{u_{i}}[q]=\widehat{\mathbf{U}}_{u_{i}}[q] \widehat{\boldsymbol{\Sigma}}_{u_{i}}[q]\left[\widehat{\mathbf{V}}_{u_{i}}^{1}[q] \widehat{\mathbf{V}}_{u_{i}}^{0}[q]\right]^{H},
$$

in order to obtain the first $\hat{L}_{i}[q]$ right singular vectors $\widehat{\mathbf{V}}_{u_{i}}^{1}[q]$, where $\widehat{L}_{i}[q]=\operatorname{rank}\left(\mathbf{G}_{i}[q]\right)$, used to compute the orthogonalization process in step (II.a) for each iteration $i>1$. Finally, in step (II.d), in order to reduce the computational complexity of the algorithm, the users whose orthogonality coefficient $\alpha_{k}$, given by,

$$
\alpha_{k}=\frac{1}{N_{c}} \sum_{q=1}^{N_{c}} \frac{\left\|\mathbf{H}_{k}[q] \widehat{\mathbf{V}}_{u_{i}}^{1}[q]\right\|_{\mathrm{F}}^{2}}{\left\|\mathbf{H}_{k}[q]\right\|_{F}^{2}\left\|\widehat{\mathbf{V}}_{u_{i}}^{1}[q]\right\|_{\mathrm{F}}^{2}}
$$

exceeds a certain threshold $\alpha$, are discarded from the total user pool. Note that $\alpha$ is a small positive value used to update the set of orthogonal users and to avoid loss in the sum-rate capacity. Hence, the selection of optimal value of $\alpha$ becomes crucial. Its selection will be discussed using simulation in Section V. The user selection process stops when the number of users in the active subset $\mathcal{U}$ equals the maximum number $K_{\max }$ or the list of remaining users is empty. Once the users have been chosen, the BD algorithm described in Section III is used to compute the precoding matrices for the selected users.

\section{NUMERICAL RESULTS}

The simulations consider the use of parameters currently found in the latest WLAN standard IEEE 802.11n and that will surely also form part of the forthcoming IEEE 802.11ac norm. The system has been configured to operate at $5.25 \mathrm{GHz}$ carrier frequency on a bandwidth of $W=20 \mathrm{MHz}$ with $N_{c}=64$ subcarriers. The channel profile used to generate the frequency-selective channel responses correspond to profiles B (residential) from channel models developed within the IEEE 802.11n standard [15]. The BS is assumed to operate with either $N_{T}=4$ and $N_{T}=8$ transmit antennas while the number of receive antennas at each mobile station has been fixed to $N_{R}=2$. Unless otherwise specified, the BS is assumed to transmit with total power $P_{T}$ to ensure that every subcarrier, on average, operates with an $S N R[q]=10 \mathrm{~dB}$.

In Fig. 2 we plot the sum-rate capacity, obtained using (23), under the proposed MUS-BD algorithm as a function of the orthogonality parameter $\alpha$. The plots are obtained by averaging over 1000 independent channel realizations. The total number of users in the cell has been fixed respectively to 10,50 and 100 . It is observed that, the orthogonality parameter $\alpha$ is mainly driven by the number of transmit antennas and the number of active users in the cell. Note that, smaller thresholds result in small user subset at each step of the algorithm, which in turn lowers the search complexity. However, too small threshold incurs throughput loss due to the reduced multiuser diversity gain. Nevertheless, if it is too large, non-orthogonal users are selected and the channel gains are reduced. Hence, it is very important to find the optimal value of $\alpha$ that yield sum-rate capacity close to that with exhaustive search method. In this figure, we can see that optimal values decrease as the number of users grows. For $N_{T}=4$, (see Fig. 1.a), optimal

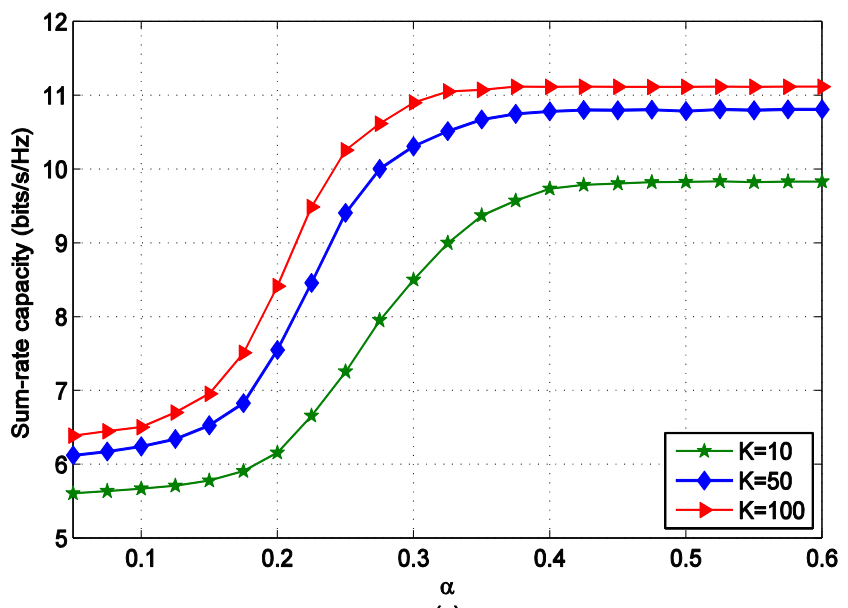

(a)

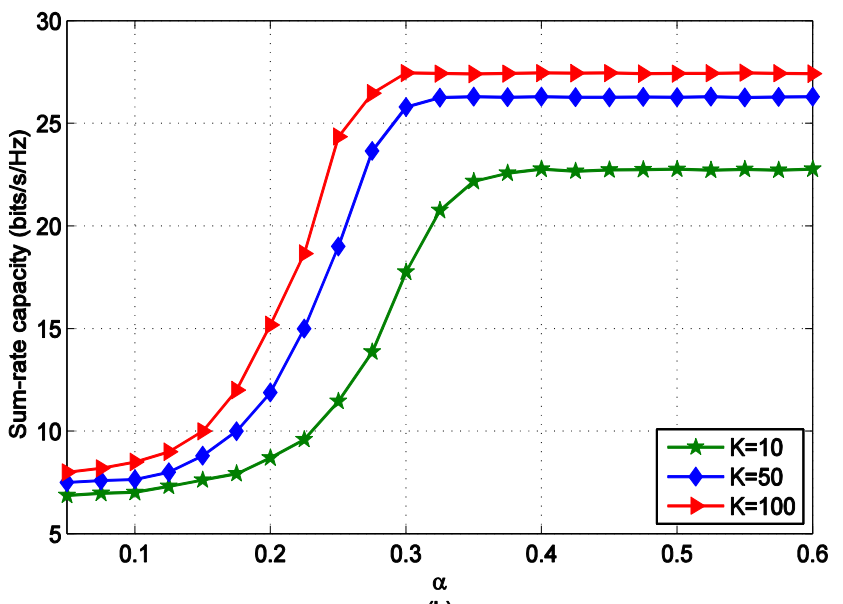

(b)

Fig.2. Sum-rate capacity vs. $\alpha . S N R[q]=10 \mathrm{~dB}$. (a) $N_{T}=4, N_{R}=2, K_{\max }=2$. (b) $N_{T}=8, N_{R}=2, K_{\max }=4$

threshold which maximizes the sum-rate capacity are in the range $[0.35-0.45]$. However, for large $N_{T}$ (e.g. $N_{T}=8$ in Fig. 1.b), this trend changes slightly and thus the optimal threshold that results in maximum throughput are in the range $[0.3-0.4]$. For the rest of simulations optimal value $\alpha$, which maximizes the sum-rate capacity, has been considered.

Fig. 3 plots the sum-rate capacity of MUS-BD, optimal scheduling and conventional opportunistic multicarrier TDMA (opp-MC-TDMA) [16] versus the number of users in the cell. In conventional opp-MC-TDMA scheme, the BS selects the single user experiencing the best channel realization at each time slot that will be allocated all the spectrum and power resources. It can be observed that, as the number of users and transmit antennas become larger, more simultaneously selected users will be found by the proposed algorithm and thus the system sum-rate capacity will be improved. Both MUS-BD and optimal scheduling algorithms manage to achieve more than $65 \%$ and $200 \%$ increase in sum-rate capacity for $N_{T}=4$ (see Fig. 1.a) and $N_{T}=8$ (see Fig. 1.b), respectively, compared to conventional opp-MC-TDMA technique. Moreover, the 
proposed MUS-BD algorithm performs very close to the optimal scheduling algorithm with much lower computational

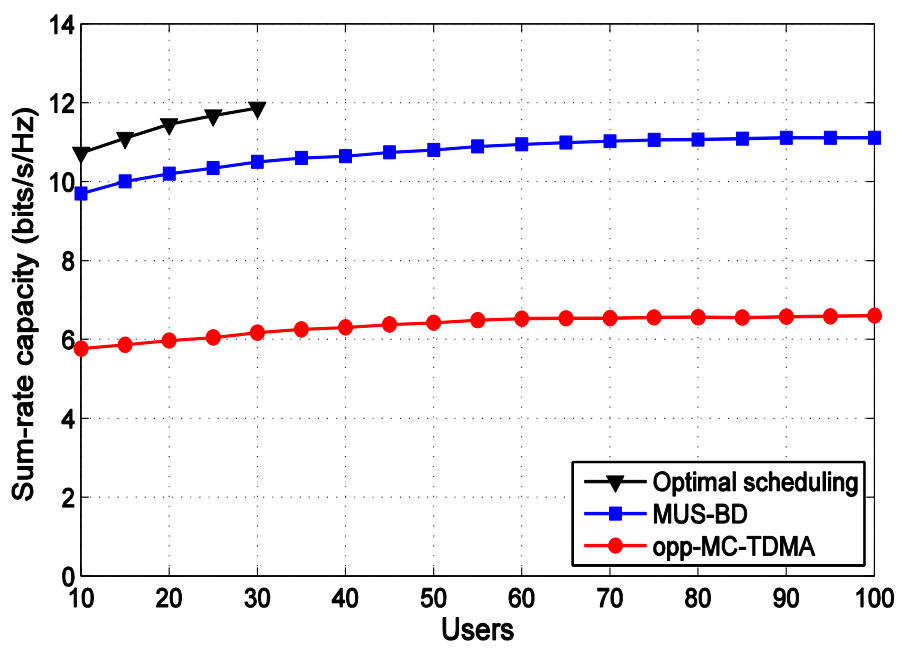

(a)

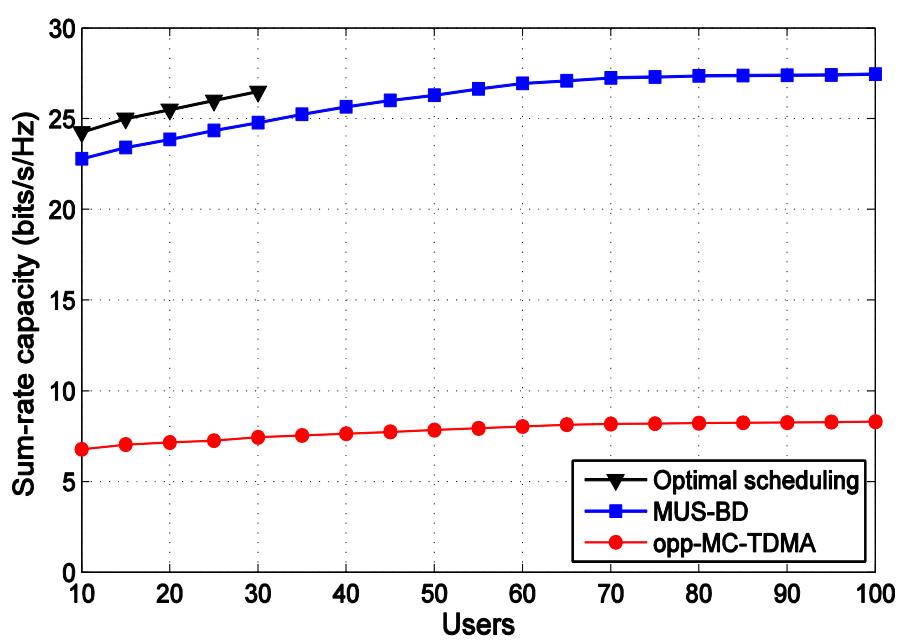

(b)

Fig.3. Sum-rate capacity vs. number of users $K . S N R[q]=10 \mathrm{~dB}$. (a) $N_{T}=4, N_{R}=2, K_{\max }=2$. (b) $N_{T}=8, N_{R}=2, K_{\max }=4$.

complexity. Note that, for optimal user selection scheme, it was only feasible to compute values for up to $K=30$ users in the cell due to the high computational complexity requirements.

Finally, Fig. 4 compares the sum-rate capacity of MUS-BD, optimal scheduling and opp-MC-TDMA as a function of the $S N R$. In this scenario the total number of users in the cell has been fixed to $K=10$ and the number of transmit antennas at BS to $N_{T}=4$. It can be seen that in the low $S N R$ regime, e.g. $S N R[q]=0 \mathrm{~dB}$, the performance of all schemes are very close to each other because fewer users are scheduled to obtain the larger transmit diversity. However, at high SNR regime, both MUS-BD and optimal scheduling schemes tend to select the maximum number of users that can be supported simultaneously by BS and thus the gain in sum-rate capacity gap is noticeable compared to the opp-MC-TDMA technique. Moreover, the proposed MUS-BD algorithm achieves more than $90 \%$ of the sum-rate capacity offered by the optimal scheduling method with much lower computational complexity.

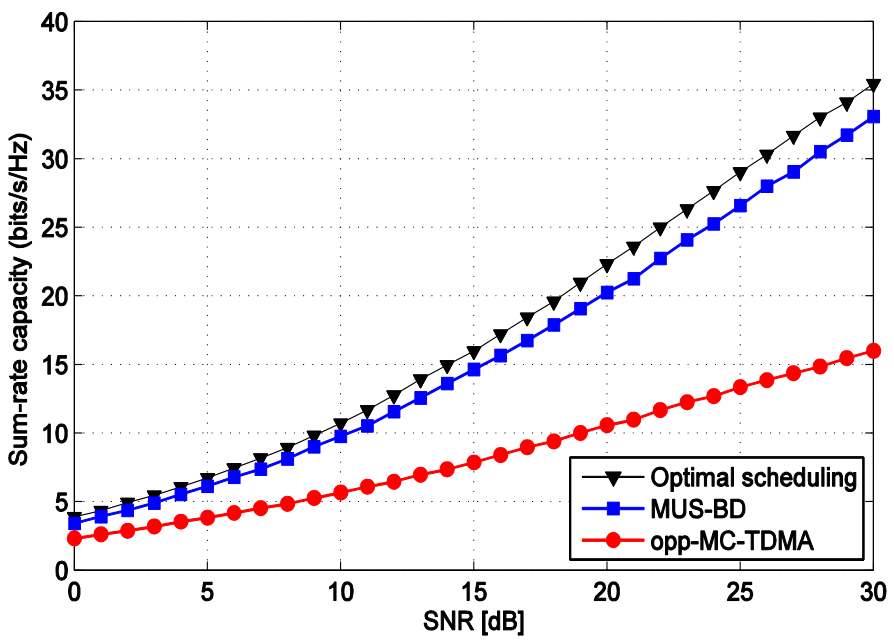

Fig.4. Sum-rate capacity vs. $S N R . K=10, N_{T}=4, N_{R}=2, K_{\max }=2$.

\section{CONCLUSIONS}

This paper has introduced a suboptimal user selection algorithm for multiuser MIMO-OFDM systems employing Block Diagonalization. Its goal is to select a subset of users to maximize the total sum-rate capacity while keeping the complexity low. The proposed scheme is composed of a user selection step that selects the users experimenting good channel realizations, and a subcarrier-specific precoder based on BD designed to pre-eliminate the multiuser interference. Simulation results have shown that the proposed scheme achieves a very significant increase in sum-rate capacity compared to conventional opportunistic MC-TDMA technique and performs close to the optimal scheduling method with much lower computational complexity.

The user selection algorithm proposed in this paper requires complete channel knowledge at the transmitter and, furthermore, it did not consider any of the fairness issues MU-MIMO brings along. Further work will address both issues by considering partial channel knowledge at the BS and introducing mechanisms to improve fairness among users.

\section{ACKNOWLEDGEMENT}

The authors would like to thank the editor and the anonymous reviewers, whose careful consideration of the manuscript improved the presentation of the material.

REFERENCES

[1] E.Telatar, "Capacity of multi-antenna Gaussian channels," Eur. Trans. Telecommun., vol. 10, no. 6, pp. 585-595, Nov. 1999.

[2] G. Foschini and M. Gans, "On limits of wireless communications in a fading environment when using multiple antennas," Wireless Personal Communications, vol. 6, pp. 311-335, Mar 1998.

[3] R. van Nee and R. Prasad, OFDM for wireless multimedia communications Artech House, 2000.

[4] A. J. Paulraj, D. A. Gore, R. U. Nabar, and H. Bolcskei, "An overview of MIMO communications a key to gigabit wireless," Proceedings of the IEEE, vol. 92, no. 2, pp. 198-218, 2004. 
[5] D. Gesbert, M. Kountouris, R. Heath, C.-B. Chae, and T. Salzer, "Shifting the MIMO paradigm," IEEE Sig. Proces. Mag., vol. 24, no. 5, Sep. 2007.

[6] A. Scaglione, P. Stoica, S. Barbarossa, G. Giannakis, and H. Sampath, "Optimal designs for space-time linear precoders and decoders," IEEE Trans. on Signal Processing, vol. 6, pp. 311-335, Mar. 1998.

[7] M. Costa, "Writing on dirty paper," IEEE Trans. Info. Theory, vol. 29, pp. 439-441, May. 1983.

[8] N. Jindal and A. Goldsmith," "Dirty-paper coding versus tdma for mimo broadcast channels," IEEE Trans. on Information Theory, vol. 51, no. 5, pp. 1783-1794, May 2005.

[9] Q. Spencer, A. Swindlehurst, and M. Haardt, "Zero-forcing methods for downlink spatial multiplexing in multiuser MIMO channels," IEEE Trans. on Signal Processing, vol. 52, no. 2, Feb. 2004.

[10] Zukang Shen; Runhua Chen; Andrews, J.G.; Heath, R.W.; Evans, B.L., "Low complexity user selection algorithms for multiuser mimo systems with block diagonalization," Signal Processing, IEEE Transactions on, vol. 54, no. 9, p. 3658,3663, Sep 2006.

[11] M. Eslami and W. A. Krzymien, "Efficient transmission technique for mimo-ofdm broadcast channels with limited feedback," in Spread Spectrum Techniques and Applications, 2008. ISSSTA '08. IEEE 10th International Symposium on, aug. 2008, pp. $237-241$.

[12] T. Yoo and A. Goldsmith, "On the optimality of multiantenna broadcast scheduling using zero-forcing beamforming," IEEE Journal on Selected Areas in Communications, vol. 24, no. 3, pp. 528-541, Mar. 2006.

[13] M. Esslaoui, F. Riera-Palou, and G. Femenias, "Opportunistic multiuser MIMO for OFDM networks." in Proc. IEEE 8th Multi-Carrier Systems \& Solutions, May 2011.

[14] N. Jindal, W. Rhee, S. Vishwanath, S. A. Jafar, and A. Goldsmith, "Sum power iterative water-filling for multi-antenna gaussian broadcast channels," IEEE Trans. on Information Theory, vol. 51, no. 4, pp. 1570-1580, April 2005.

[15] J. Kermoal, L. Schumacher, K. Pedersen, P. Mogensen, and F. Frederiksen, "A stochastic MIMO radio channel model with experimental validation," IEEE JSAC, vol. 20, no. 6, pp. 1211-1226, Aug 2002.

[16] P. Viswanath, D. Tse, and R. Laroia, "Opportunistic beamforming using dumb antennas,” IEEE Trans. on Inf. Theory, vol. 48, no. 6, Jun. 2002.

\section{AUTHORS PROFILE}

Mounir Esslaoui received the Master degree in Electronics and Telecommunications from Abdelmalek Essaadi University, Tetouan, Morocco in 2008. He is currently working towards the Ph.D. degree in information and Communication sciences at Abdelmalek Essaadi University. He was an exchanged $\mathrm{Ph} . \mathrm{D}$. student at Mobile Communications Group laboratory, University of the Balearic Islands, Spain, from 2009 till 2012. His research is currently focused on the forthcoming generation of wireless communication networks with particular emphasis on multiuser MIMO and multicarrier systems.

Mohamed Essaaidi received the Ph.D. degree in Electrical Engineering in 1997 from Abdelmalek Essaadi University, Tetouan, Morocco. He is the current Director of National College of IT (ENSIAS) of Mohammed 5th Souissi University, Rabat, Morocco and he was a Professor of Electrical and Computer Engineering at Abdelmalek Essaadi University, Morocco from 1993 till 2011. He is an IEEE Senior Member, the founder and Chairman of the IEEE Morocco Section, founder of IEEE Computer \& Communication Societies Joint Morocco Chapter, Founder and Chair of IEEE Antennas and Propagation Society and Microwave Theory and Techniques Society Morocco Joint Chapter and founder of IEEE Education Society Morocco Chapter. He has been also the founding Director of the Morocco Office of Arab Science and Technology Foundation, ASTF (2006-2009) and the Coordinator of ASTF RD\&I Network of Electro-Technology since 2006. He has also founded several IEEE Student Branches in different Moroccan universities and engineering schools.

He has authored and co-authored 5 books and more than 120 papers in international refereed journals and conferences in the field of Electrical, Information and Communication Technologies. $\mathrm{He}$ has been the Editor-in-Chief of International Journal on Information and Communication Technologies, Serial Publications, India since 2007. He is also an active member of the editorial boards of several IEEE and other indexed international journals in the field of information and communication technologies.

Dr. Essaaidi holds four patents on antennas for very high data rate UWB and multi-band wireless communication systems and high resolution medical imaging systems. Furthermore, he has co-organized / been involved in the juries of several national and international competitions aiming at fostering research, development and innovation such as Moroccan Engineers Week 2006, 2007, "Made in Morocco", Arab Science and Technology Foundation (ASTF) "Made in Arabia" Competitions in 2007 and 2009, Qatar Foundation Stars of Science 2010 and Intel Science Competition 2011. 